\title{
Receive Counseling
}

National Cancer Institute

\section{Source}

National Cancer Institute. Receive Counseling. NCI Thesaurus. Code C128524.

The patient must receive counseling from a healthcare provider. The counseling may cover a specific topic, use a specific material, or both. 